\title{
Mechatronic fingernail with static and dynamic force sensing
}

\author{
Risto Kõiva*, Tobias Schwank, Guillaume Walck, Robert Haschke and Helge J. Ritter
}

\begin{abstract}
Our fingernails help us to accomplish a variety of manual tasks, but surprisingly only a few robotic hands are equipped with nails. In this paper, we present a sensorized fingernail for mechatronic hands that can capture static and dynamic interaction forces with the nail. Over the course of several iterations, we have developed a very compact working prototype that fits together with our previously developed multi-cell tactile fingertip sensor into the cavity of the distal phalange of a human-sized robotic hand. We present the construction details, list the key performance characteristics and demonstrate an example application of finding the end of an adhesive tape roll using the signals captured by the sensors integrated in the nail. We conclude with a discussion about improvement ideas for future versions.
\end{abstract}

\section{INTRODUCTION}

Only very few robotic hands have been equipped with artificial nails [1]-[3], which is surprising, as the fingernail plays an important role in human grasping and fine manipulation [4]. The nails help us to accomplish many everyday tasks, like pick, peel, scratch and dig and they become very hard or even infeasible without nails.

Human hands, especially the fingertips, have one of the highest concentrations of mechanoreceptors [5], the organs that sense mechanical pressure and distortions occurring at our skin. We argue that the high sensitivity and the diversity of our touch receptors are the key factors for humans being able to grasp and manipulate objects with hands so effortlessly. Recent advances in state-of-the-art tactile sensing in robotic hands [6]-[12] are a significant step towards robotic manual intelligence. Most previous attempts at sensorizing the robotic hands and fingers developed robotic skin or measured forces and torques at finger, hand or wrist joints.

Only a single experiment is known to us, where artificial fingernails were sensorized - in [13] Sinapov et al. present rudimentary fingernails, cut out of plastic sheet, that were equipped with three-axis digital accelerometers and were used to classify the surfaces contacted from the highfrequency signal induced during surface scratching. To the best of our knowledge, the work was never implemented on anthropomorphic dexterous mechatronic hands and further development was not pursued by the authors.

Our design goal was to develop a miniature fingernail capable of vibratory as well as static 3D contact force sensing. The vibratory data aims to capture surface properties

This work was supported by project FAMULA of the Cluster of Excellence Cognitive Interaction Technology CITEC (EXC 277) at Bielefeld University, which is funded by the German Research Foundation (DFG).

${ }^{*}$ R. Kõiva, T. Schwank, G. Walck, R. Haschke work for and H.J. Ritter leads the Neuroinformatics Group, Center of Excellence Cognitive Interaction Technology, Bielefeld University, 33619 Bielefeld, Germany. Correspondence: risto.koiva@uni-bielefeld.de when scratching, similar to the work presented in [13], but also to be used in concert with the data from other nail sensors to detect very small protrusions or edges, as we will demonstrate in an example application of detecting an end of a tape roll [Sec. IV]. The sensors measuring static contact force direction \& magnitude primarily target contact feedback for tactile servoing and tactile exploration tasks [14]. The hardware development was not stipulated with specific parameters to be reached or a specific task to be solved, instead our goal was to come up with well working multi-purpose tool in the very limited space available.

\section{DETAILS OF CONSTRUCTION}

As our graspLab ${ }^{1}$ is equipped with multiple Shadow Robot Hands [2], we chose to develop our fingernail prototype for this specific hardware. Nevertheless, the small size and the human-like shape of the fingernail should enable to integrate it on other robotic or prosthetic hands where the required space is available, thanks to a single screw attachment and a standardized $\mathrm{I}^{2} \mathrm{C}$ interface.

Our latest iteration is a mechatronic fingernail for robotic hands, equipped with a multitude of sensors capturing static and dynamic interaction forces. The fingernail has two main parts - a rigid-flex printed-circuit-board (PCB) populated with digital sensors in surface-mount-devices (SMD) packages as the base; and the nail part, $\mathrm{CNC}$ milled out of white

${ }^{1}$ https://ni.www.techfak.uni-bielefeld.de/labs

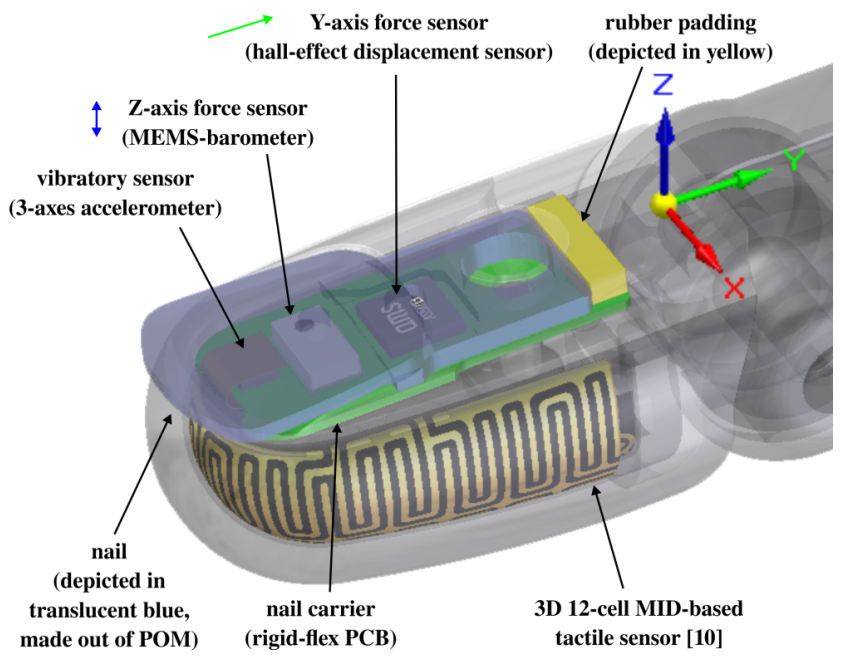

Fig. 1. Novel fingernail sensor with multiple interaction force sensing channels shares the distal phalange cavity space with our previously developed MID-based 3D shaped 12-taxel fingertip force sensor [10]. 
polyoxymethylene (POM) thermoplastic. Fig. 1 depicts the fingernail construction in a translucent CAD rendering and defines the sensor's coordinate axes used throughout this paper. The plastic nail is able to move minimally along the positive $\mathrm{Y}$ axis, held in place (when not pushed) at it's most distal position using a rubber padding located behind the nail, acting as a spring (shown in yellow in Fig. 1).

The electronic components in our fingernail are connected to a shared digital $\mathrm{I}^{2} \mathrm{C}$ bus, necessitating only a 4-wire electrical connection $\left(\mathrm{I}^{2} \mathrm{C}\right.$ clock (SCL), data (SDA), ground reference and voltage supply). As the digitization of the measured signals is performed directly in the fingertip, the design is robust against electrical noise.

\section{A. Y-AXIS FORCE SENSING}

For measuring the fingernail deflection along $\mathrm{Y}$ axis, we opted for AMS AS5013 EasyPoint magnetic position sensor chip due to its small size of only $4 \times 4 \times 0.55 \mathrm{~mm}$ and high sensitivity to detect small deflections. This Hall-effect-IC is measuring the movement of the nail with the help of a $\varnothing 2$ $\mathrm{mm} \times 0.8 \mathrm{~mm}$ neodymium magnet of type AS5000-MA2H-1, press-fit into a milled pocket in the nail. The nail can move approx. up to $0.5 \mathrm{~mm}$ from its idle, most distal, position. The output of the sensor-IC is a signed 8-bit value, whereas the raw sensor center value (zero) is roughly in the middle of the full nail displacement range.

\section{B. Z-AXIS FORCE SENSING}

The vertical push and pull forces exerted on the nail are measured using a Freescale/NXP MPL115A2 absolute digital barometer chip. Inspired by Tenzer et al. [15], using under-pressure we filled the pressure sensor and the gap area between the sensor and the nail with silicon (Relicon Religel by HellermannTyton). The silicon filling propagates the mechanical forces occurring on the nail into the MEMS membrane inside the sensor chip, originally designed to measure barometric pressure changes. This setup achieves very high sensitivity, as presented in detail in Sec. III.

In a previous iteration of the fingernail, we experimented using the absolute digital pressure sensor NXP MPL3115A2, a successor of MPL115A2 with considerably higher internal ADC resolution of 24-bits vs. 10-bits. The orifice of MPL3115A2 is although only $\varnothing 0.5 \mathrm{~mm}$ vs. $\varnothing 1 \mathrm{~mm}$ on the MPL115A2. This considerably smaller opening made silicon injection more difficult, resulting in mediocre force sensing due to bad transmission of nail forces onto the MEMS membrane, reasoning our preference for the older chip.

\section{VIBRATORY SENSING}

The dynamic high-frequency vibrations, e.g. occurring when scratching surfaces, are captured in the fingernail with a Bosch-Sensortec BMA255 triaxial accelerometer chip in a tiny $2 \times 2 \times 0.95 \mathrm{~mm}$ package. The BMA255 is soldered on the distal flex-part of the PCB and adhered using a thin double sided tape to the underside of the nail. The flexible PCB connection separates the accelerometer from the fixed base, which avoids damping of the vibratory signals.

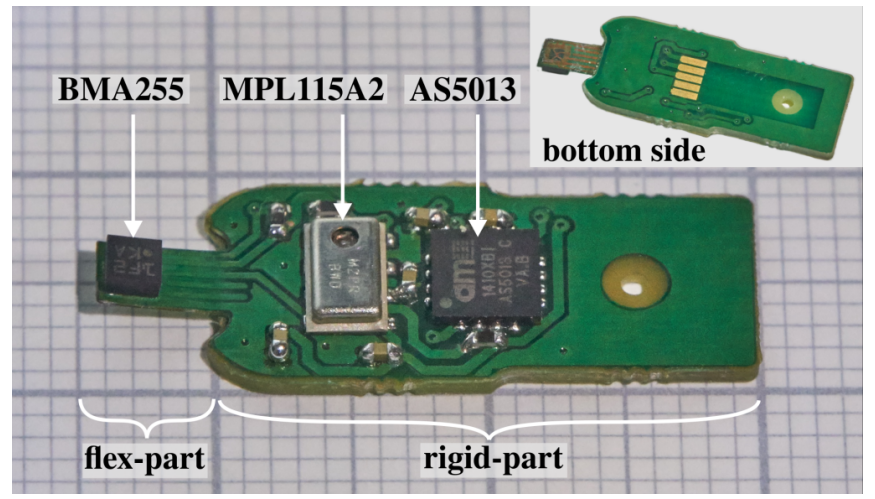

Fig. 2. The PCB of the fingernail with a BMA255 triaxial acceleration sensor, a MPL115A2 barometer (Z-axis) and a AS5013 Hall-effect sensor (Y-axes). The upper right inset shows the bottom side of the PCB with contact pads for power and $\mathrm{I}^{2} \mathrm{C}$ communication. For size comparison, the PCB was photographed on top of $1 \mathrm{~mm}$ grid graph paper.

Instead of using an accelerometer to sample the nail vibrations, we previously also experimented with a wideband MEMS microphone. Although the microphone worked amazingly well for capturing vibratory patterns occurring on the nail, it also picked up sound (voices) from the near vicinity of the fingertip and was rejected for privacy reasons.

\section{COMPONENT LAYOUT \& DATA READOUT}

Fig. 2 shows the PCB with populated sensor chips before the assembly of the plastic nail and the following silicon pouring process. To help to grasp the scale of the PCB, a millimeter paper was used as the background in the image.

For evaluation purposes, a digital interface based on a Teensy 3.2 board serves as a gateway between the nail sensors on $\mathrm{I}^{2} \mathrm{C}$ bus and the PC. The gathered data from the sensors is streamed out over a serial port emulation protocol to ROS node at the PC side, which publishes messages over separate topics for each type of signals, augmented with a timestamp for recording and post-processing purposes.

\section{EVALUATION}

We performed three experiments with the fingernail prototype - first, a detection threshold sensitivity measurement; second, a characterization of the sensor over the respective measurement ranges of the axes; and third, a validation of the vibratory sensor output when exposed to various frequencies and frequency combinations during excitation.

\section{A. Sensor detection threshold}

Due to significant differences in the sensitivity of the $\mathrm{Y}$ and $\mathrm{Z}$ axes, two different strategies were necessary to measure the detection thresholds.

For Z-axis, we resorted to a set of precision weights, placed in steps of $50 \mathrm{mg}$ onto the nail [Fig. 3], while the sensor raw output was monitored for a change. Nail pulling (positive Z-axis direction) was measured with the finger turned upside down and the weights attached with thin double sided tape onto the nail. The weight of the tape was measured and accounted for in the calculations. The 


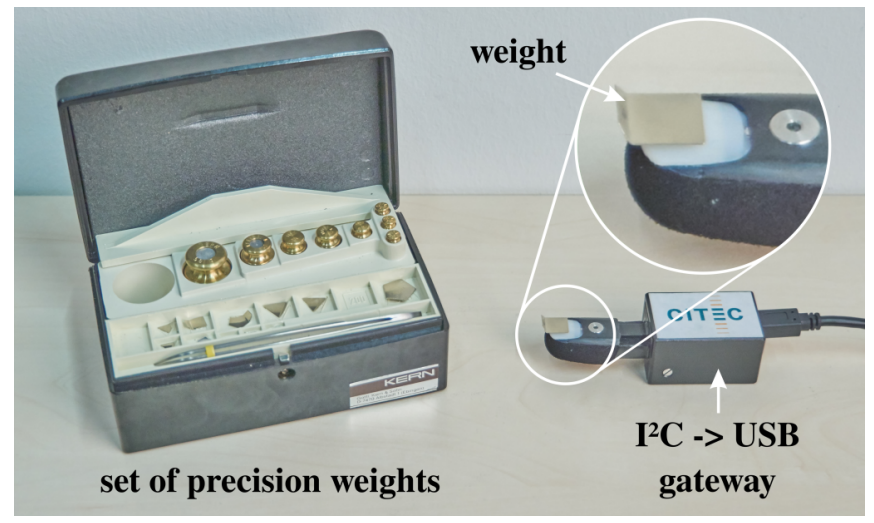

Fig. 3. A set of precision weights in steps of $50 \mathrm{mg}$ were used to evaluate the threshold of the Z-axis force sensor. For measuring the push force (negative $\mathrm{Z}$ direction), the weights were stacked onto the nail, as pictured, while simultaneously the sensor readout was observed on the connected PC. For fingernail pulling measurements (positive $\mathrm{Z}$ direction), the sensor was turned upside-down and the weights were adhered using thin double sided tape onto the nail.

detection threshold was experimentally found over 10 trials to be $4.4 \mathrm{mN} \pm 0.7 \mathrm{mN}$ for pushing (Z-) and $8.8 \mathrm{mN} \pm 0.8$ $\mathrm{mN}$ for pulling $(\mathrm{Z}+)$. We highlight that the contact detection threshold never exceeded a value of $10 \mathrm{mN}$. In the pushing (Z-) direction, the fingernail was able to reliably detect all contacts higher than very low $6 \mathrm{mN}$.

To measure the detection threshold along the Y-axis, we built a custom CNC measurement bench [Fig. 4]. An industrial calibrated force sensor (ME-Meßsysteme $\mathrm{GmbH}$ $\mathrm{KD} 45$ ) with a $\pm 10 \mathrm{~N}$ range attached on a linear springloaded mount was used as a reference sensor. Its signal, amplified by force sensor amplifier (GSV-1L from the same manufacturer), was connected to an analog input of a Labjack U6 DAQ device. The force was increased in small increments and the raw fingernail sensor output observed for a change. The threshold for detection on the Y-axis was found to be $1.17 \mathrm{~N} \pm 0.02 \mathrm{~N}$ (averaged over 10 measurements). In a future version, we consider experimenting with softer rubber variations for the padding in order to further decrease the contact detection threshold of the $\mathrm{Y}$ axis, although there is a trade-off with measurement range.

\section{B. Characteristic curves}

To characterize the fingernail sensor axes, we used the same CNC measurement bench as explained before [Fig. 4]. While recording the raw sensor signal and the signal from the reference sensor, the force was increased by displacing the vertical axis of the measurement bench in small increments. We evaluated also the sensor response to pauses of various lengths in-between each step. A single trial lasted between 35 seconds and 17 minutes, depending on the chosen pause duration and the direction (push or pull) measured.

Fig. 5 displays the characteristics of the Z-axis sensor for push and pull forces. The asymmetry of the saturation level between these opposite forces comes from the silicon between the nail and the sensor. The silicon does not adhere strongly on POM, which leads to a weaker force transmission

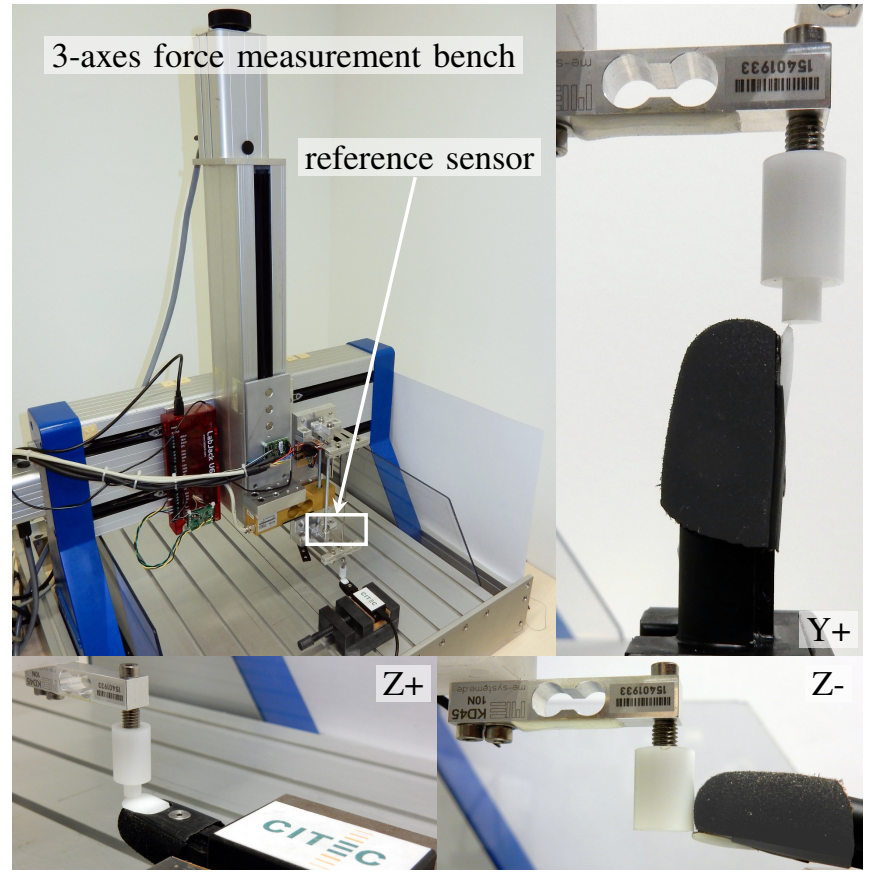

Fig. 4. Fingernail sensor characterizations were performed with a custom built 3-axes CNC controlled measurement bench. An industrial calibrated strain gauge based force sensor, with $\pm 10 \mathrm{~N}$ nominal range, was used as a reference sensor. During measurements, the reference sensor signal and the raw signal of the sensor under test were simultaneously captured. The three inserts display the different orientations the fingernail sensor was tested.

when pulling. This limitation implicitly saturates measurements of pull forces at a lower threshold than for push forces. We found the relaxation time to influence the extent of the sensor range, whereas the characteristic curve retained mostly it's shape and hysteresis. Almost linear characteristic was observed in the $[-0.5,0.5] \mathrm{N}$ range. The raw output at zero force was not recorded long enough to show the return to starting point after relaxation, which explains why the push and pull characteristic curves are not connected.

The characteristic of the Y-axis hall effect sensor is shown in Fig. 6. It is rather linear, but has a significant hysteresis, which likely comes from high friction between the plastic nail and the surrounding fingertip mounting part. The sensor produced repeatable values in the linear part with a variation of 3 LSB over 10 trials. The noise level is only 1 LSB.

\section{Vibratory sensor evaluation}

To validate the function of the vibratory sensor, we used a computer controlled shaker that we held in contact with the fingernail. A set of different sinusoidal waveforms at $75 \mathrm{~Hz}, 120 \mathrm{~Hz}, 200 \mathrm{~Hz}, 300 \mathrm{~Hz}, 400 \mathrm{~Hz}$ and a signal with overlapping sinusoidal waveforms at $120 \mathrm{~Hz}+200$ $\mathrm{Hz}$ were generated. While these signals were successively played on the shaker, we observed a live FFT output to validate that the captured signal matched the input signal frequencies. As can also be seen in the accompanying video, the frequency peaks matched the stimulus within $1 \mathrm{~Hz}$, indicating well behaving sensory system over the full range. Fig. 7 displays the experimental setup. 


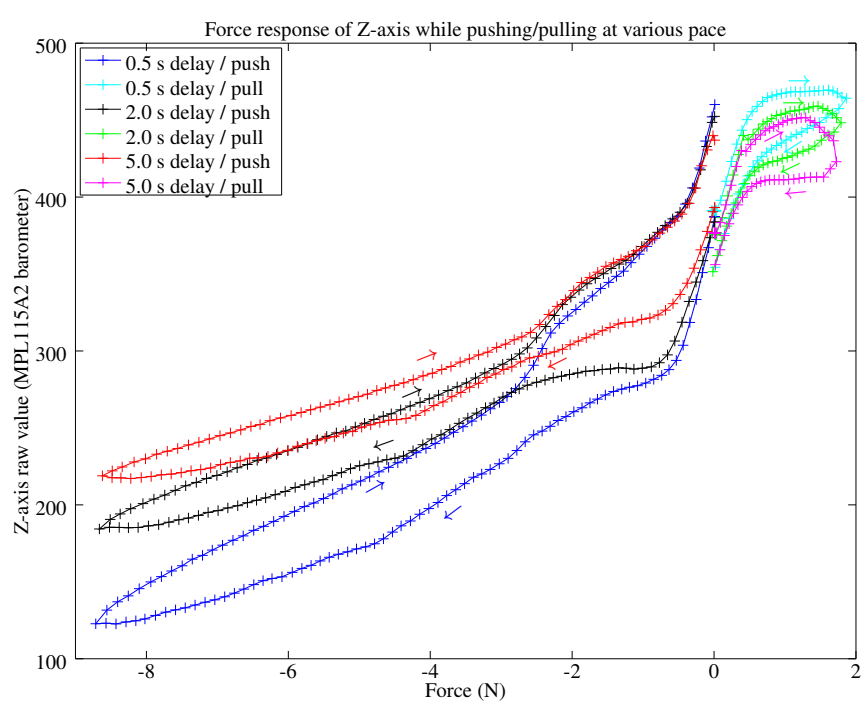

Fig. 5. Combined plot of 6 measurement curves of the Z-axis sensor - 3 different delays for push (Z-) as well as pull (Z+) direction. The displayed push and pull curves don't precisely touch due to silicon slow retraction and us not waiting longer between the measurements.

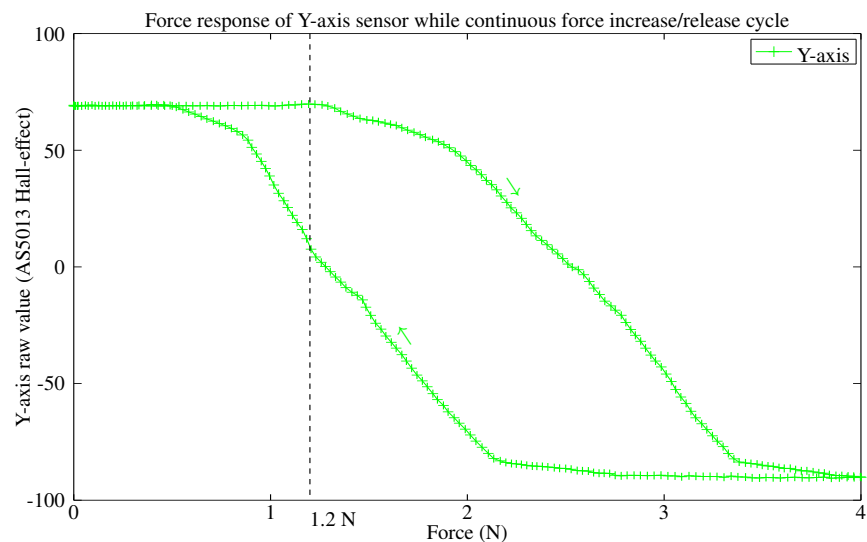

Fig. 6. Y-axis force sensor characteristics of a single trial (from 10), and the detection threshold (vertical dashed-line) measured over 10 trials.

Acquisition delays were measured from a contact event until its detection in the sensor data. The accelerometer (2 $\mathrm{kHz}$ ), detects the contact in the next sample $86 \%$ of the time, but never later than $1.5 \mathrm{~ms}$, whereas the barometer $(200 \mathrm{~Hz})$ detects it $88 \%$ of the time in less than $10 \mathrm{~ms}$, but never later than $20 \mathrm{~ms}$, which is sufficient for a tactile servoing loop.

\section{EXAMPLE APPLICATION}

An initial test comparing the vibratory sensor of the fingernail to the multi-cell tactile fingertip was performed in a task of material classification from surface scratching as done in [13]. A deep neural network classifier, which has proven to solve hard tactile perception tasks [16], processes the vibratory data of the nail and shows classification accuracy of $75 \%$ for 8 types of material on the validation set, when the same classifier reached only $50 \%$ correct results using the tactile data. This suggests that the rigid nail better transmits vibrations to the sensor than the foam of the tactile sensor.

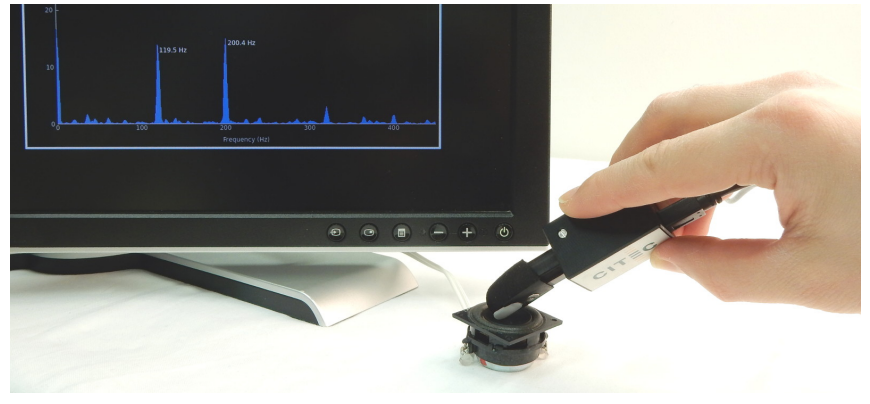

Fig. 7. The fingernail held against a computer controlled shaker (loudspeaker) generating a dual tone sinusoidal waveforms at $120 \mathrm{~Hz}$ and 200 $\mathrm{Hz}$, while a live FFT of the accelerometer Z-axis signal is displayed on screen. The peaks are detected at the awaited frequencies.

To further test the multi-sensor fingernail, we chose a real life scenario that is challenging even for humans - finding the end of an adhesive tape roll. We found this task to be difficult to solve with a robotic system using only computer vision, especially when lighting conditions and observation angle are not perfect. No compact robust tactile sensor that can fit into human-sized robotic hands is known to us to have a high enough sensitivity to localize the end of a tape roll. We set up an experiment to find out if our fingernail prototype can master this ambitious task.

\section{A. Experimental setup}

We built a test rig to slide a roll of tape at a constant speed under our fingernail prototype [Fig. 8]. Although assembled

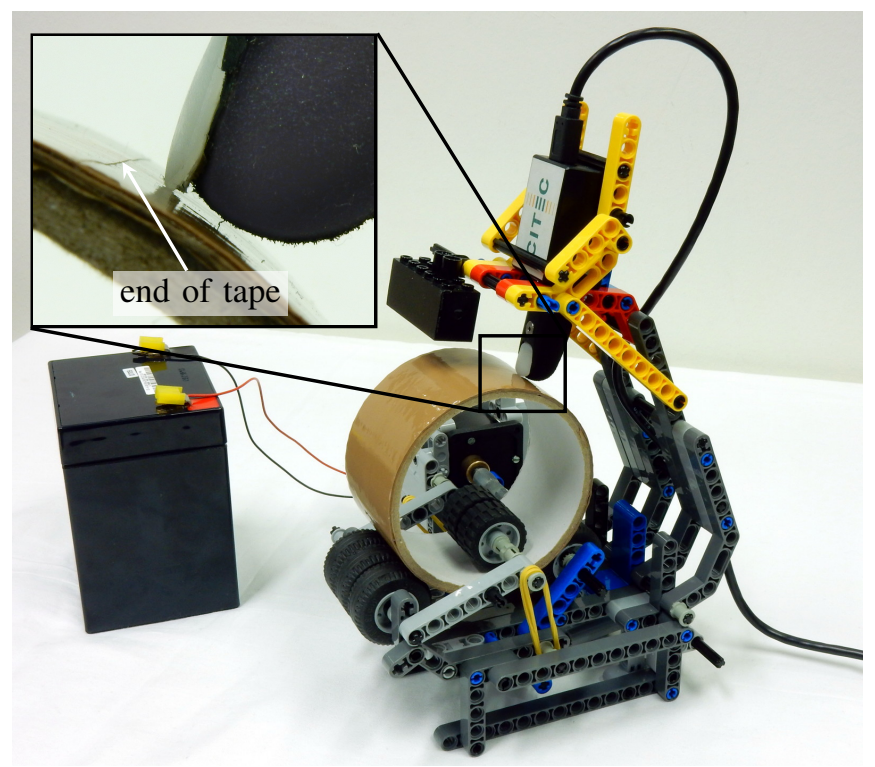

Fig. 8. Test rig for evaluating the fingernail sensor by finding the end of tape. For this, the contraption rotates a tape-roll at constant speed under the fingernail, while the signals of nail sensors are captured. The test setup is composed of 3 sets of rollers, one set is actuated by a DC motor, one set is spring-loaded to constrain the position of the tape-roll, and the last set's position is adjustable to fit for various roll diameters. The mechatronic finger is held by a swing arm that permits adjusting the contact force and the angle of contact. The upper left inset depicts the relative thickness of the barely visible edge to be detected in comparison to the fingernail size. 


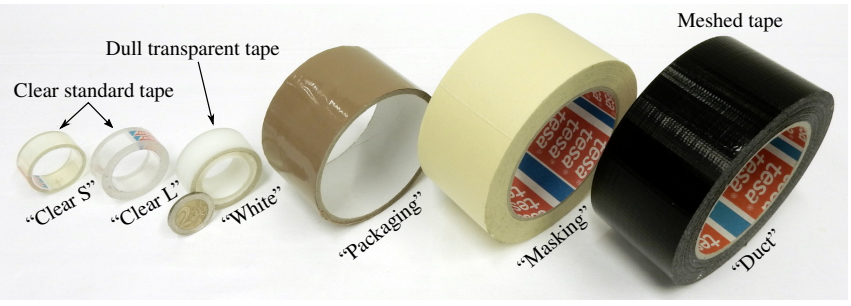

Fig. 9. The adhesive tape rolls considered in the experiment. For size comparison, we included a 2 euro coin in the image.

out of toy parts, the rig is stable, accommodates for various tape diameters and ensures stable rolling without significant lateral movements. The contact angle was chosen to be as close as possible to the surface normal, but at the same time avoiding contact with fingertip areas other than the nail. A static force was applied implicitly along the contact direction of the fingernail by using the weight of the fingertip itself plus additional weights on the lever. The linear scratching speed was identical for all tapes (due to driving wheels on the outer surface) and chosen experimentally to avoid resonance effects of the fingertip jumping over the surface due to small imperfections, and thus to ensure contact between the fingernail and the tape roll throughout full rotation.

We tested various types of common tape rolls [Fig. 9], covering different sizes, thickness and surface roughnesses. The masking tape and the meshed "duct" tape had the roughest surfaces in the test, where on the latter the regular mesh can easily be felt by a human touch. The other test candidates had smooth surfaces. The thinnest tapes tested were the brown packaging tape and the dull transparent tape.

We tried finding an edge of the tape roll in both directions - hitting the edge of tape, as well as "falling" from the edge. Experimentally we found the tactile sensation to be different for the two cases as shown later.

Three types of signals were recorded during four revolutions of the rolls: the vibratory force was captured at 900 $\mathrm{Hz}$ along $\mathrm{Y}$ and $\mathrm{Z}$ axes, and the force along the $\mathrm{Z}$-axis was recorded at $150 \mathrm{~Hz}$ with the barometric sensor, all of them expected to signal hitting a rising edge.

\section{B. Experimental results}

Fig. 10 shows the sensor output of accelerometer and barometer sensors while making 4 rotations with a Clear $L$ tape. There are four strong regularly-spaced peaks corresponding to the revolutions of that roll on the test rig. The amplitude of the peaks is more than 3 times higher than the noise level of the signals, providing an easy detection of the end of a tape roll using a simple threshold-based classifier. The Clear $L$ tape has a rather thick film but a smooth surface, creating a nice edge while not producing significant noise in the signal. All rolls produced consistent repetition of peaks over the four revolutions, but showed different amplitudes of the peaks and the noise. Fig. 11 summarizes these results by showing the sensor response in the vicinity of a single peak for all types of considered tape.
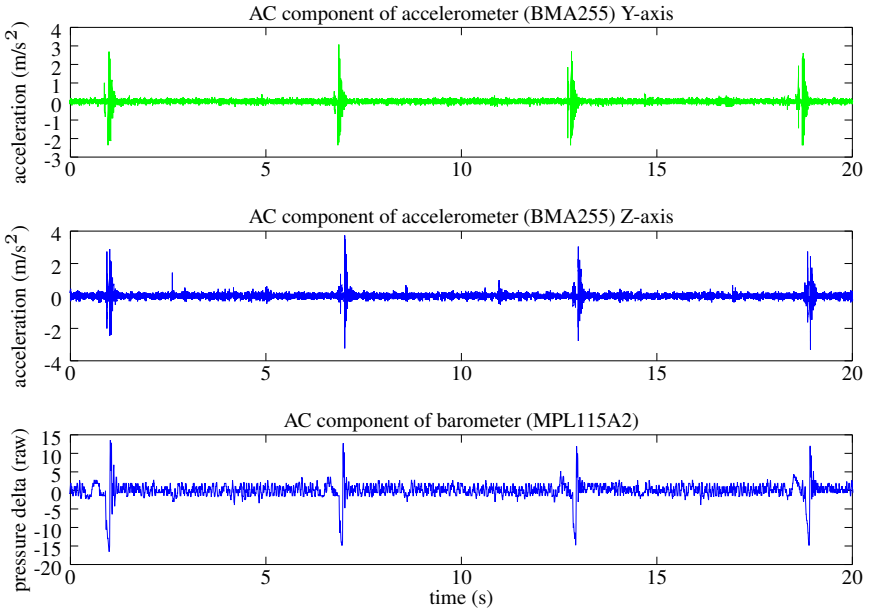

Fig. 10. Comparison of 3 fingernail sensor signals (top: accelerometer Yaxis, middle: accelerometer Z-axis, bottom: barometer) while sliding over a Clear $L$ tape. The duration spans over 4 rotations at a constant linear speed, and shows 4 clear peaks on each signal corresponding to the fingernail encountering the end of a tape roll with a rising edge. The force used to press the nail against the tape was roughly $0.7 \mathrm{~N}$.

We observed that the tapes with rougher surfaces cause higher levels of noise during rotation. For the masking tape tested, only the signal of accelerometer Z-axis was able to capture a detectable peak. The surface roughness of other considered tapes was lower and thus produced clearer peaks at every edge in more than one sensor output, thus increasing the confidence in detecting the end of a tape roll.

From the two contact forces tested $(0.25 \mathrm{~N}$ and $0.7 \mathrm{~N})$, the higher force reduced the noise on the signal of the vibratory sensor, probably due to the increased inertia of the swing arm that damped the scratching vibrations. At the same time it
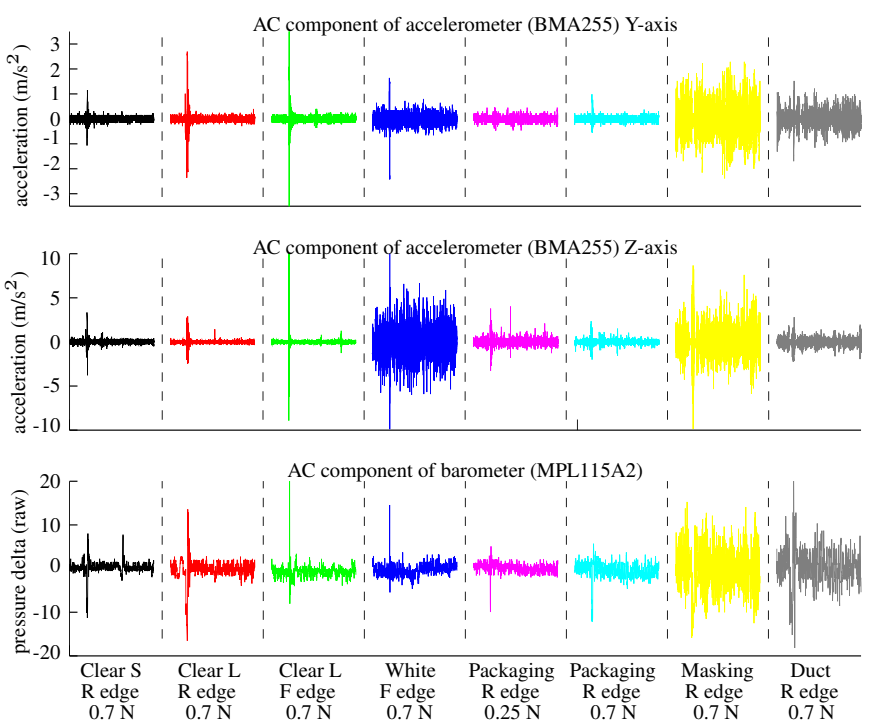

Fig. 11. Plots of 3 fingernail sensor signals (top: accelerometer Y-axis, middle: accelerometer Z-axis, bottom: barometer) while sliding with the fingernail over our selection of adhesive tapes. The plots use the same scale and show a single peak per type of roll, corresponding to the fingernail encountering the end of a tape roll. 
also increased the peak height for the sensors during hitting the end of tape (accelerometer Y-axis and barometer). Hence, in a realistic scenario with the sensor embedded in a robotic hand, a scratching force with roughly defined value should be maintained (our study showed $0.7 \mathrm{~N}$ to work best), to ensure that a peak in the signal can be robustly detected.

Two types of tape were rolled also in the opposite direction to produce a falling edge instead of a rising edge. This change generated a considerably stronger peak in the accelerometer signal along the Y-axis than the peak measured with the same tape during a rising edge, more prone to agitate the sensor on the Z-axis. This effect comes from the step generating a height change in the appropriate accelerometer axis. The difference of signals between a rising and a falling edge response is interesting, and can be used to detect the direction the tape end should be peeled off.

Overall, the measurements along the Z-axis were sensitive enough and produced a good peak-to-noise ratio for finding the end of a tape-roll for most types tested using basic threshold classifiers. Exploiting more advanced classifiers, e.g. deep neural networks, we are confident that the remaining types of tape can be successfully handled as well. The vibratory sensor is more sensitive to surface roughness, possibly missing the peak covered by the noise, but can be used as a complementary source of data.

\section{CONCLUSION \& FUTURE WORK}

We presented a very compact mechatronic fingernail to be integrated in robotic or prosthetic hands. The embedded sensors can capture static and dynamic interaction forces, opening new interesting usage scenarios, either when detecting the contact or providing force feedback for control loops driving the hand and arm joints. The developed sensor is very sensitive in the Z-axis, in both, push and pull, directions. No more than $10 \mathrm{mN}$ is required for reliable contact detection. The Y-axis can sense contacts starting from $1.17 \mathrm{~N}$, which enables collision detection, but could also measure the hardness of some materials. The vibratory sensor is sensitive enough to produce different signals when scratching various surface types. Using the sensors in concert, we demonstrated the fingernail capability of reliably detecting the end of a tape-roll, a task that is even hard for humans to perform.

We will next, integrate the developed sensor in our lab's Shadow Robot Hand. The next generation MID based distal fingertip tactile sensor, currently under development, will integrate the $\mathrm{I}^{2} \mathrm{C}$ interface to sample the sensors of the nail. It will stream, together with the distal joint tactile sensor data, the nail data onto the Shadow Robot Hand's internal SPI bus, where the information gets further transmitted out towards the main control unit. We hope the fingernail sensor to increase the robustness of our robotic hand-arm system when performing every-day tasks common for humans, such as detecting and opening lids, switching buried buttons, distinguishing surface textures to name a few. We are also excited to re-experiment the task of finding an end of a tape roll with robotic hands, especially when guiding the roll in front of the nail with another hand.

Plans are afoot to redesign the shape of the mechanical nail mounting parts and change the shape of the rubber padding in order for the nail to move in $\mathrm{X}-\mathrm{Y}$ plane instead of just along Y-axis. The electronic design is already prepared for this, as the AS5013 hall-effect sensor can sample magnetic displacement in 2 dimensions. We will also experiment with rubber materials to possibly increase the sensitivity of Y-axis force detection. Due to the previous success with MEMSmicrophone, we plan to test out a dual microphone setup to cancel out ambient sounds from the nail vibrations.

\section{REFERENCES}

[1] K. Murakami and T. Hasegawa, "Novel fingertip equipped with soft skin and hard nail for dexterous multi-fingered robotic manipulation," in IEEE International Conference on Robotics and Automation (ICRA), vol. 1, Sept 2003, pp. 708-713.

[2] "Shadow Dexterous Hand," http://www.shadowrobot.com/products/ dexterous-hand/, Cited on July 2018.

[3] K. V. D. S. Chathuranga, V. A. Ho, and S. Hirai, "A Bio-mimetic Fingertip That Detects Force and Vibration Modalities and its Application to Surface Identification," in IEEE International Conference on Robotics and Biomimetics (ROBIO), Dec 2012, pp. 575-581.

[4] S. A. Mascaro and H. H. Asada, "Understanding of fingernail-bone interaction and fingertip hemodynamics for fingernail sensor design," in Haptic Interfaces for Virtual Environment and Teleoperator Systems (HAPTICS), 2002, pp. 106-113.

[5] R. S. Johansson and J. R. Flanagan, "Coding and use of tactile signals from the fingertips in object manipulation tasks," Nature reviews. Neuroscience, vol. 10, pp. 345-359, May 2009.

[6] R. S. Dahiya, G. Metta, M. Valle, and G. Sandini, "Tactile Sensing From Humans to Humanoids," IEEE Transactions on Robotics, vol. 26 , no. 1, pp. 1-20, Feb. 2010.

[7] J. Butterfass, M. Grebenstein, H. Liu, and G. Hirzinger, "DLR-Hand II: next generation of a dextrous robot hand," in IEEE International Conference on Robotics and Automation (ICRA), vol. 1, Korea, 2001, pp. 109-114.

[8] A. Schmitz, P. Maiolino, M. Maggiali, L. Natale, G. Cannata, and G. Metta, "Methods and Technologies for the Implementation of Large-Scale Robot Tactile Sensors," IEEE Transactions on Robotics, vol. 27 , no. 3, pp. 389-400, 2011.

[9] "SynTouch BioTac robotic fingertip tactile sensor," http://www. syntouchllc.com/Products/BioTac/, Cited on July 2018.

[10] R. Kõiva, M. Zenker, C. Schürmann, R. Haschke, and H. J. Ritter, "A highly sensitive 3D-shaped tactile sensor," in IEEE/ASME International Conference on Advanced Intelligent Mechatronics (AIM), Wollongong, Australia, July 2013, pp. 1084-1089.

[11] G. Büscher, M. Meier, G. Walck, R. Haschke, and H. J. Ritter "Augmenting curved robot surfaces with soft tactile skin," in IEEE/RSJ International Conference on Intelligent Robots and Systems, Sept 2015, pp. 1514-1519.

[12] L. Zou, C. Ge, Z. J. Wang, E. Cretu, and X. Li, "Novel Tactile Sensor Technology and Smart Tactile Sensing Systems: A Review," Sensors, vol. 17 , no. 11, 2017.

[13] J. Sinapov, V. Sukhoy, R. Sahai, and A. Stoytchev, "Vibrotactile Recognition and Categorization of Surfaces by a Humanoid Robot," IEEE Transactions on Robotics, vol. 27, no. 3, pp. 488-497, 2011.

[14] Q. Li, R. Haschke, and H. Ritter, "A Visuo-Tactile Control Framework for Manipulation and Exploration of Unknown Objects," in IEEE-RAS 15th International Conference on Humanoid Robots (Humanoids), 2015.

[15] Y. Tenzer, L. P. Jentoft, and R. D. Howe, "The Feel of MEMS Barometers: Inexpensive and Easily Customized Tactile Array Sensors," IEEE Robotics Automation Magazine, vol. 21, no. 3, pp. 89-95, Sept 2014.

[16] M. Meier, F. Patzelt, R. Haschke, and H. Ritter, "Tactile Convolutional Networks for Online Slip and Rotation Detection," in International Conference on Artificial Neural Networks (ICANN), 2016. 